\title{
Characterization of turbulence stability through the identification of multifractional Brownian motions
}

\author{
K. C. Lee \\ Department of Industrial Engineering, Hanyang University, Seoul, Korea
}

Correspondence to: K. C. Lee (skylee@ehanyang.ac.kr)

Received: 27 August 2012 - Revised: 4 December 2012 - Accepted: 6 January 2013 - Published: 6 February 2013

\begin{abstract}
Multifractional Brownian motions have become popular as flexible models in describing real-life signals of high-frequency features in geoscience, microeconomics, and turbulence, to name a few. The time-changing Hurst exponent, which describes regularity levels depending on time measurements, and variance, which relates to an energy level, are two parameters that characterize multifractional Brownian motions. This research suggests a combined method of estimating the time-changing Hurst exponent and variance using the local variation of sampled paths of signals. The method consists of two phases: initially estimating global variance and then accurately estimating the time-changing Hurst exponent. A simulation study shows its performance in estimation of the parameters. The proposed method is applied to characterization of atmospheric stability in which descriptive statistics from the estimated time-changing Hurst exponent and variance classify stable atmosphere flows from unstable ones.
\end{abstract}

\section{Introduction}

It has been observed that a wide range of complex signals in nature are characterized by seemingly irregular behavior varying in time and exhibiting different levels of magnitude. Examples of such irregular signals in time are abundant in medicine, economics, and geoscience, to list a few. Such signals can be effectively modeled by multifractional Brownian motions $(\mathrm{mBm})$. As a generalization of fractional Brownian motions ( $\mathrm{fBm}$ ), multifractional Brownian motions describe both regularity attributes varying depending on time measurements and variance levels describing orders of energy magnitude. The regularity attribute, also called the Hurst exponent, expresses the strength of statistical similarity at many different frequencies and is extended to vary with time, to model realistic situations occurring in various fields.

With such a perspective, a few approaches have been proposed to model multifractional Brownian motions. LévyVéhel and Peltier (1995) proposed a mean average approach. Benassi et al. (1998) introduced a spectral approach. The proposed models represent $\mathrm{mBm}$ as a Gaussian process $W(t)$ with a covariance function involving the Hurst exponent $H$ by a function of time, $H(t)$, and variance parameter $C$. The process $W$ is well defined, or square-integrable, if function $H(t)$ is continuous enough in a Hölderian sense, and its variance at time $t$ is given by $\operatorname{Var}[W(t)]=C^{2} t^{H(t)}$ parameterized by variance level $C$ and the Hurst exponent (Cohen, 1999). The time-changing Hurst exponent $H(t)$ characterizes the path regularity of process $W$ at time $t$, since sample paths near $t$ with small $H(t)$, close to 0 , are space-filling and highly irregular, while paths with large $H(t)$, close to 1 , are very smooth. The variance constant $C$ determines the energy level of the process.

Several approaches have been proposed to estimate the time-changing Hurst exponent $H(t)$ and variance $C$ from sample paths of $\mathrm{mBm}$ signals. Benassi et al. (1998) examined estimation of a continuously differentiable $H(t)$ without the direct estimation of $C$. Fhima et al. (2011) used the increment ratio statistic method for estimation of $H(t)$ only. For estimation of $H(t)$, Benassi et al. (2000) and Fhima et al. (2011) adopted the approach of relaxing the continuous condition of $H(t)$ by piecewise constants. For an overview of estimating constant $H(t)$, the reader is also referred to Beran (1994), including various statistical methods, or Bardet and Bertrand (2007), emphasizing wavelet contexts. The estimation of both $H(t)$ and variance parameter $C$ has received little attention from the statistics community, while $C$ is mostly treated as a nuisance parameter. Coeurjolly (2005) used a 
local version of quadratic variations and proposed a class of estimator of $H(t)$ and $C$ at each local sample path. When a signal is modeled with $\mathrm{mBm}$, the estimation of $H(t)$ can be improved by estimating $C$ accurately from covariance structures involving both $H(t)$ and $C$.

The objective of this paper is to propose a procedure for accurately estimating $H(t)$ and $C$ given a sampled path of $\mathrm{mBm}$ process $W$. This paper uses discrete filtering techniques for $\mathrm{mBm}$, further calculating $k$-th empirical moments of the filtered signals related to $H(t)$ and $C$. Then the following two phases are adopted. The first phase estimates $H(t)$ and $C$ for each local sample path and leads to a global estimator of $C$. The second phase adjusts estimated $H(t)$ with the global estimator of $C$ from the previous phase. The adopted two-phased approach results in an estimation of $C$ insensitive to many sample paths, global to process $W$, and thus an accurate estimation of $H(t)$.

The suggested approach is applied to turbulence signals of air velocity and temperature. Turbulence stability for atmospheric flows is determined by Monin-Obukhov lengths from the similarity theory and related to the convection of the air (Monin and Obukhov, 1954; Obukhov, 1971; Stull, 1988). Recent evidence shows that atmospheric turbulence is anisotropic and requires different scaling exponents, which is related to the Hurst exponent, depending on directions (Lovejoy et al., 2007, 2010), and also that multifractality and generalized scale invariance analysis reveals rich information on scaling exponents (Lovejoy et al., 2009; Schertzer and Lovejoy, 2011). In this paper, turbulence signals of longitudinal, lateral, and vertical velocities and temperature are identified with $\mathrm{mBm}$ by estimating $H(t)$ and $C$ (Ghil, 1985; Wanliss and Dobias, 2007). Descriptive statistics generated from estimated $H(t)$ and $C$ are used in classifying stable flows from unstable ones.

The paper is organized as follows. Section 2 introduces computation of $k$-th empirical moments of filtered signals and explains the proposed two-phased procedure of estimating the parameters. Section 3 discusses numerical simulations and shows the benefit of the proposed approach. Section 4 presents applications of the proposed approach to air turbulence signals. Section 5 concludes this paper.

\section{Method}

Let us consider a discretized sample path from a Gaussian process $W(i / N)$, a mBm signal, for $i=1, \ldots, N$, with Hurst function $H(t)$ and variance parameter $C$. The spectral representation of $W$ is given by

$W(t)=C \sqrt{K(2 H(t)) / 2} \int_{\mathbb{R}} \frac{e^{\mathbf{i} t \lambda}-1}{|\lambda|^{H(t)+1 / 2}} d B(\lambda)$,

where $C$ is a constant scale (variance) parameter and $B$ the standard Brownian motion (Benassi et al., 1998). Similarly, the mean average approach of $W$ is given by

$$
\begin{aligned}
& W(t)=C \frac{\sqrt{\pi K(2 H(t))}}{\Gamma(H(t)+1 / 2)} \\
& \int_{\mathbb{R}}\left\{|t-s|^{H(t)-1 / 2} \mathbf{1}_{]-\infty, t]}(s)-|s|^{H(t)-1 / 2} \mathbf{1}_{]-\infty, 0]}(s)\right\} d B(s),
\end{aligned}
$$

where $B$ is the standard Brownian motion (Lévy-Véhel and Peltier, 1995). The terms "process" and "signal" will be interchangeably used in referring to observed sample paths of a random process. It is worth noting that observed variables in the atmosphere, when collected for all time measurements, are not normally distributed, but that the empirical evidence shows the necessity of interactive random processes consisting of a Hurst exponent (Tuck, 2010). The Hurst function $H(t)$ is assumed to be continuous enough in a Hölderian sense as in Cohen (1999). The covariance function of $W$ for $t, v \in[0,1]$ is given by

$$
\begin{aligned}
\mathcal{E}[W(t) W(v)] & =\frac{C^{2}}{2} \frac{\sqrt{K(2 H(t)) K(2 H(v))}}{K(H(t)+H(v))} \\
\left\{|t|^{H(t)+H(v)}+|v|^{H(t)+H(v)}-|t-v|^{H(t)+H(v)}\right\}, &
\end{aligned}
$$

where $K(x)=\Gamma(x+1) \sin (x \pi / 2) / \pi$ (Ayache et al., 2000; Coeurjolly, 2005). From the covariance function, we have $\mathcal{E}\left[W(t)^{2}\right]=C^{2} t^{2 H(t)}$, and consequently $\operatorname{Var}[W(1)]=C^{2}$. In this sense, $C$ is called the variance level of the process. It is clear to see the process $W(t)$ is not weakly stationary, since the covariance function does not depend on $t-v$ only. The estimation of $C$ is nontrivial because of the dependence structure of $C$ and $H(t)$ from the covariance function; that is to say, the sample variance of a sample path does not lead to the direct expression of $C$. For example, for a given sample path from $t_{1}$ to $t_{n}, \mathcal{E}\left[\sum_{k=1}^{n} W\left(t_{k}\right)^{2}\right]=C^{2} \sum_{k=1}^{n} t^{2 H\left(t_{k}\right)}$ depends upon not only $C$ but also $H\left(t_{k}\right)$ for all $k$.

In order to weaken the dependence structure in $W(t)$, a differencing filter a of length $l+1$ and order $p>1$ (the number of vanishing moments) is applied. Filter $\mathbf{a}$ is defined by its taps, $\left(a_{0}, \ldots, a_{l}\right)$, such that

$$
\sum_{q=0}^{l} a_{q} q^{i}=0, i=0, \ldots, p-1, \text { and } \sum_{q=0}^{l} a_{q} q^{i} \neq 0, i=p .
$$

For instance, possible choices for filter $\mathbf{a}$ are shown in Table 1. For difference filters, the length $l+1$ is equal to the order $p$ plus one, while for Daubechies filters it is equal to two times the order $p$. Furthermore, high-pass wavelet filters with a different number of vanishing moments corresponding to orthogonal wavelets such as Daubechies, Symlet, and Coiflet wavelets are also possible. A detailed discussion of wavelet filters can be found in Daubechies (1992) and Vidakovic (1999). 
Table 1. Examples for filter a are shown.

\begin{tabular}{lccc}
\hline \multicolumn{1}{c}{ name } & filter a & order $p$ & length $l+1$ \\
\hline difference $(d 2)$ & $(1,-2,1)$ & 2 & 3 \\
difference $(d 3)$ & $(1,-3,3,1)$ & 3 & 4 \\
Daubechies $(D 2)$ & $(0.48296,-0.83652,0.22414,0.12941)$ & 2 & 4 \\
Daubechies $(D 3)$ & $(0.33267,-0.80689,0.45988,0.13501,-0.085441$, & 3 & 6 \\
Symlet $(S 4)$ & $-0.035226)$ & 4 & 8 \\
Symlet $(S 5)$ & $(0.027333,-0.029519,-0.039134,-0.1994,0.72341$, & 5 & 10 \\
& $-0.63398,0.016602,0.17533,-0.021102,-0.019539)$ & & \\
\hline
\end{tabular}

Let us also introduce $\mathbf{a}^{(m)}$ based on filter $\mathbf{a}$, the filter defined by

$a_{i}^{(m)}= \begin{cases}a_{i / m}, & i / m \text { is an integer }, \\ 0, & \text { otherwise. }\end{cases}$

Notice that $\mathbf{a}^{(m)}$, the filter a dilated $m$ times, captures a resolution at a low frequency, corresponding to a coarse space, as $m$ increases. For $m=1$, it captures the finest level of detail. For example, $\mathbf{a}^{(1)}=\mathbf{a}$ by definition, and for second-order difference filter $\mathbf{d} 2, \mathbf{a}=(1,-2,1), \mathbf{a}^{(2)}$ becomes $(1,0,-2,0,1)$.

Let $V_{\mathbf{a}^{(m)}}$ be a process of local variation consisting of $W$ filtered by $\mathbf{a}^{(m)}$, that is,

$V_{\mathbf{a}^{(m)}}\left(\frac{j}{N}\right)=\sum_{q=0}^{m l} a_{q}^{(m)} W\left(\frac{j-q}{N}\right)$, for $j=m l+1, \ldots, N$.

For example, when $\mathbf{a}^{(1)}=(1,-2,1)$ of order 2 is applied, $V_{\mathbf{a}^{(1)}}$ represents the second-order differences of $W$. It is possible to show that the filtered signal $V_{\mathbf{a}^{(m)}}(t)$ is weakly stationary, so the filtering by $\mathbf{a}^{(m)}$ breaks the dependence structure between observations. See Appendix A for proof. Additionally, $V_{\mathbf{a}^{(m)}}(t)$ converges to a locally stationary Gaussian process with the following variance structure: for $j / N$ in a neighborhood of $t$, denoted by $v_{t}$, of a sufficiently small size,

$\operatorname{Var}\left[V_{\mathbf{a}^{(m)}}\left(\frac{j}{N}\right)\right]=\frac{C^{2} m^{2 H(t)}}{N^{2 H(t)}} \pi_{\mathbf{a}, H(t)}$,

where $\pi_{\mathbf{a}, h}=-\frac{1}{2} \sum_{q, q^{\prime}=0}^{l} a_{q} a_{q^{\prime}}\left|q-q^{\prime}\right|^{2 h}$. Then the $k$-th absolute moment of $V_{\mathbf{a}^{(m)}}$ follows by normalizing $V_{\mathbf{a}^{(m)}}$ by the standard deviation, $\sigma=\sqrt{\operatorname{Var}\left[V_{\mathbf{a}^{(m)}}\left(\frac{j}{N}\right)\right]}$ :

$$
\begin{aligned}
\mathcal{E}\left[\left|V_{\mathbf{a}^{(m)}}\left(\frac{j}{N}\right)\right|^{k}\right] & =\mathcal{E}\left[\left|\frac{\left.\left.V_{\mathbf{a}^{(m)}\left(\frac{j}{N}\right)}\right|^{k}\right] \sigma^{k}}{\sigma}\right|^{k}\right. \\
& =E_{k}\left(\frac{C m^{H(t)}}{N^{H(t)}} \pi_{\mathbf{a}, H(t)}^{1 / 2}\right)^{k}
\end{aligned}
$$

where $\frac{V_{\mathbf{a}^{(m)}}\left(\frac{j}{N}\right)}{\sigma} \sim N(0,1)$ and $E_{k}$ is the $k$-th absolute moment of the standard Gaussian variable given by $E_{k}=$ $2^{k / 2} \Gamma((k+1) / 2) / \sqrt{\pi}$ for $k>0$ (Papoulis, 1991). Accordingly, the log of the $k$-th absolute moment is derived as follows:

$$
\begin{aligned}
\log \mathcal{E}\left[\left|V_{\mathbf{a}^{(m)}}\left(\frac{j}{N}\right)\right|^{k}\right] \\
=\log E_{k}+k \log C+\frac{k}{2} \log \pi_{\mathbf{a}, H(t)}+k H(t) \log \frac{m}{N} .
\end{aligned}
$$

Now, the $\log$ of the $k$-th empirical absolute moment near $v_{t}$, denoted by $S_{\mathbf{a}^{(m)}, k}(t)$, is introduced to estimate $\log \mathcal{E}\left[\left|V_{\mathbf{a}^{(m)}}\left(\frac{j}{N}\right)\right|^{k}\right]$ by the moment matching approach:

$S_{\mathbf{a}^{(m)}, k}(t)=\log \left(\frac{1}{|v(t)|} \sum_{j \in v(t)}\left|V_{\mathbf{a}^{(m)}}\left(\frac{j}{N}\right)\right|^{k}\right)$.

Observably, the right-hand side of Eq. (2) is linear in $H(t)$ when $\log \pi_{\mathbf{a}, H(t)}$ is ignorable. Thus, for fixed $t$ and $k$ and varying $m$, it is reasonable to consider $M$ filters $(1, \ldots, M$ for $m$ ) and to regress $L_{t}$ on $X$, where $L_{t}=\left\{S_{\mathbf{a}^{(m)}, k}(t)\right\}_{1 \leq m \leq M}$ and $X$ is the design matrix $(M \times 2)$ defined by $X=\left[\begin{array}{ll}\mathbf{1}_{M} & \mathbf{x}\end{array}\right]$ with $\mathbf{1}_{M}$ the all-ones vector $(M \times 1)$ and $\mathbf{x}=\left\{k \log \frac{m}{N}\right\}_{1 \leq m \leq M}$. That is to say,

$$
\begin{gathered}
S_{\mathbf{a}^{(m)}, k}(t) \sim \log E_{k}+k \log C+\frac{k}{2} \log \pi_{\mathbf{a}, H(t)}+k H(t) \log \frac{m}{N} \\
=\left[\begin{array}{ll}
1 & k \log \frac{m}{N}
\end{array}\right]\left[\begin{array}{c}
\theta_{t} \\
H(t)
\end{array}\right], \quad m=1, \ldots, M,
\end{gathered}
$$

where $\theta_{t}=\log E_{k}+k \log C+k / 2 \log \pi_{\mathbf{a}, H(t)}$, and $L_{t}$ is regressed with the model $\left[\begin{array}{ll}\mathbf{1}_{M} & \mathbf{x}\end{array}\right]\left[\begin{array}{ll}\theta_{t} & H(t)\end{array}\right]^{\top}$ by ordinary least squares. This procedure leads to a class of estimators of $\left[\theta_{t} H(t)\right]^{\top}$, and the least-square estimators are given as follows:

$$
\begin{aligned}
\hat{H}_{L}(t) & =\frac{\boldsymbol{A}^{\top} L_{t}}{k\|\boldsymbol{A}\|^{2}}, \\
\hat{\theta}_{t} & =\frac{\mathbf{1}_{M}^{\top} L_{t}}{M}-\frac{\boldsymbol{A}^{\top} L_{t} \sum_{j=1}^{M} \log \frac{j}{N}}{M\|\boldsymbol{A}\|^{2}},
\end{aligned}
$$


where the vector $\boldsymbol{A}(M \times 1)$ is defined by $A_{m}=\log \frac{m}{N}-$ $M^{-1} \sum_{j=1}^{M} \log \frac{j}{N}$ for $m=1, \ldots, M$. This estimator is a local version of the estimator for the Hurst exponent of $\mathrm{fBm}$ models (Coeurjolly, 2001) and an extension of the second moment result for $\mathrm{mBm}$ models to the $k$-th moments (Coeurjolly, 2005). Accordingly, the estimator of $C$, denoted by $\hat{C}_{t}$ depending on neighborhood $v_{t}$ around $t$, is derived from the definition of $\hat{\theta}_{t}$ as follows:

$\hat{C}_{t}=\pi_{\mathbf{a}, \hat{H}_{L}(t)}^{-\frac{1}{2}} \exp \left(k^{-1}\left(\hat{\theta}_{t}-\log E_{k}\right)\right)$.

Noticeably, the estimator $\hat{H}_{L}(t)$ in Eq. (4) through regression model (3) can be enhanced by using improved estimation of $C$. As the first phase, estimation of $C$ is adapted and improved by treating $\hat{C}_{t}$ as initial estimates varying by $t$ and taking the average of all estimated values $\hat{C}_{t}$ :

$\hat{C}=1 /|\Omega| \sum_{t \in \Omega} \hat{C}_{t}$,

where $\Omega$ is the index set of $t$. The adapted estimator of $C$ is denoted by $\hat{C}$. One would choose the median of $\hat{C}_{t}$ instead of the average for robust estimation. This adaptation is sensible because variance parameter $C$ is originally unvarying regardless of neighborhood position $t$ in process $W$. Accordingly, estimators of $H(t)$ at the second phase follow from the relationship between $k$-th absolute moments and $H(t)$ that is manifested in Eq. (2). The computation for $\hat{H}(t)$ is separable into each $\hat{H}\left(t_{i}\right)$ for $t_{i} \in \Omega$. In other words, for fixed $t_{i}$, a solution of $H\left(t_{i}\right)$ is given by

$$
\begin{aligned}
\hat{H}\left(t_{i}\right) & =\underset{0<h<1}{\arg \min } \sum_{m=1}^{M} \mid S_{\mathbf{a}^{(m)}, k}\left(t_{i}\right) \\
& -\left(\log E_{k}+k \log \hat{C}+\frac{k}{2} \log \pi_{\mathbf{a}, h}+k h \log \frac{m}{N}\right) \mid .
\end{aligned}
$$

Obviously, the estimator $\hat{H}(t)$ in Eq. (7) from the second phase is distinguishable from $\hat{H}_{L}(t)$ in Eq. (4), which is identical with Coeurjolly's method for $k=2$ (Coeurjolly, 2005), in that $\hat{H}(t)$ is based on globally estimated $\hat{C}$. The two estimators are compared in the following section. Numerical approaches such as the gradient descent method, the NewtonRaphson method, and the bisection method can be used for the above procedure, which is nonlinear in $h$. Because of difficulty in calculating derivatives of the objective function, the bisection method was adopted. The bisection method achieves a desired precision level, $\epsilon$, for $\hat{H}(t)$ with the number of iterations greater than $\log _{2} \epsilon^{-1}$. In other words, 10 iterations, for example, results in precision $\epsilon<0.001$, which provided a sufficient level of both computational time and precision.

\section{Simulation}

A simulation study of the performance of the suggested approach, denoted by GC (meaning globally estimated $C$ ), is

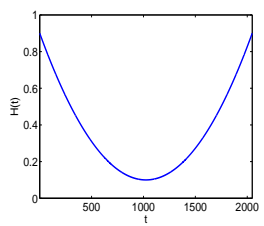

(a) U-shape $H(t)$

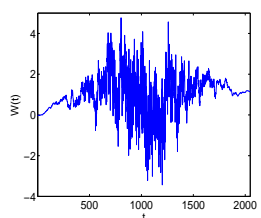

(d) $\mathrm{mBm}$ based on (a)

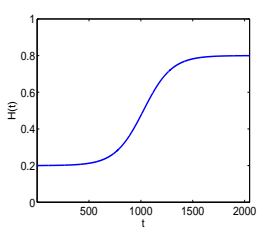

(b) logistic $H(t)$

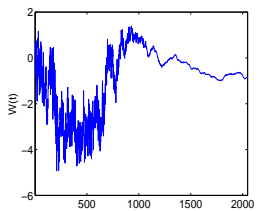

(e) $\mathrm{mBm}$ based on (b)

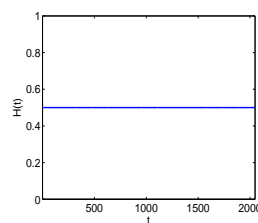

(c) flat-line $H(t)$

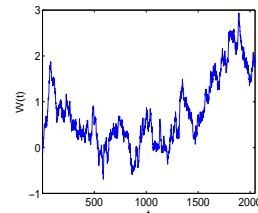

(f) $\mathrm{mBm}$ based on (c)
Fig. 1. Tested $H(t)$ functions, (a) $U$-shape, (b) logistic, and (c) flatline, are shown in the upper row. The corresponding simulated $\mathrm{mBm}$ signals with $C=3$ are shown in the lower row.

presented. Simulation is done with the "known truth" of Hurst function $H(t)$. Test functions are the $U$-shape, logistic, and flat-line functions shown in the upper row in Fig. 1; the corresponding simulated $\mathrm{mBm}$ signals are shown in the lower row. For example, the simulated signal in Fig. 1d, based on the U-shape $H(t)$ in Fig. 1a, exhibits high regularity at the starting and high irregularity in the middle as $t$ increases, ending with high regularity. The flat-line function will be used as an indicator in which the method can properly distinguish $\mathrm{fBm}$ models from $\mathrm{mBm}$ models. For the sake of comparison, we chose the estimator $\hat{H}_{L}(t)$ in Eq. (4), the method presented in Coeurjolly (2005), which is denoted by LC (meaning locally estimated $C$ ). Similarly to the GC method, the LC method estimates $H(t)$ and $C$, being based on local variations using Hermite polynomials of degree 2 . It corresponds to $k=2$ in Eq. (5) and local variance parameters. Furthermore, we included one more popular method, the wavelet-based local spectra slope, which is summarized in Gao (2007) and denoted by WV. Estimating Hurst exponents only, the method WV serves as a benchmark for the comparison with the other methods. For the detailed settings of WV, the two levels by which spectral slopes are calculated were the coarsest level of detail plus one and the finest level of detail minus one. To simulate a sample path from a $\mathrm{mBm}$, the method of Wood and Chan (1994) was used, which is exact in theory and sufficiently fast for a reasonable sample size $N$. One thousand series of length $N=2048$ and $C=3$ for each of the three functions were generated. A difference filter of order $2 d 2$, Daubechies wavelet filter of order $2 D 2$, and a Symlet wavelet filter of order $4 S 4$ were used for the three Hurst functions, respectively. For the GC method, $k$ was set to 2 , and the neighborhood size $\left|v_{t}\right|$ to 50 . The average mean squared error (MSE) was used as a performance measure to capture the difference between true $H(t)$ and estimated $\hat{H}(t)$, and empirical confidence intervals were obtained. 


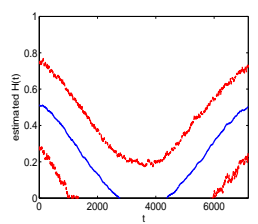

(a) WV, U-shape $\hat{H}(t)$

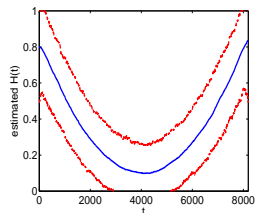

(d) LC, U-shape $\hat{H}_{L}(t)$

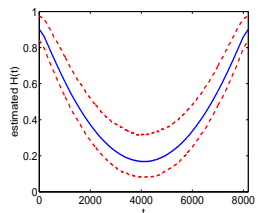

(g) $\mathrm{GC}_{1}, \mathrm{U}$-shape $\hat{H}(t)$

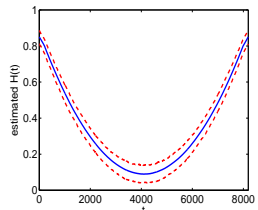

(j) $\mathrm{GC}_{2}$, U-shape $\hat{H}(t)$

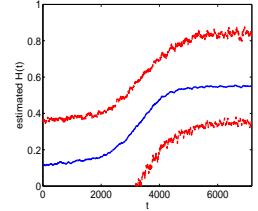

(b) WV, logistic $\hat{H}(t)$

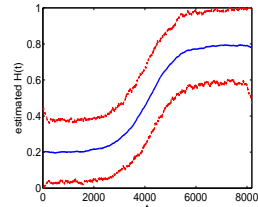

(e) LC, logistic $\hat{H}_{L}(t)$

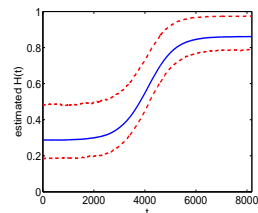

(h) $\mathrm{GC}_{1}$, logistic $\hat{H}(t)$

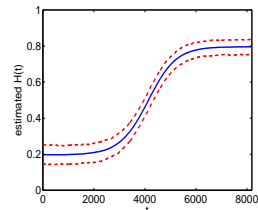

(k) $\mathrm{GC}_{2}$, logistic $\hat{H}(t)$

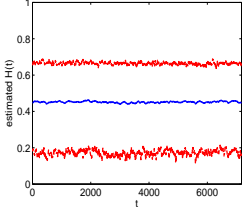

(c) WV, flat-line $\hat{H}(t)$

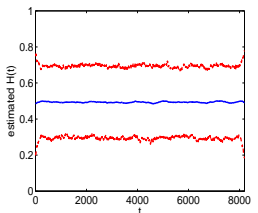

(f) LC, flat-line $\hat{H}_{L}(t)$

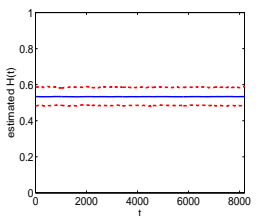

(i) $\mathrm{GC}_{1}$, flat-line $\hat{H}(t)$

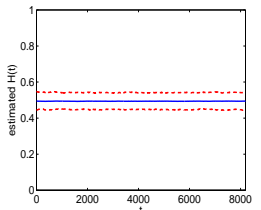

(1) $\mathrm{GC}_{2}$, flat-line $\hat{H}(t$
Fig. 2. Estimated $H(t)$ (the middle line in blue) and their empirical $95 \%$ confidence intervals (the upper and the lower dashed lines in red) for the three tested $H(t)$ functions are shown. The upper row is for the WV method, the second row $\mathrm{LC}$, the third row $\mathrm{GC}_{1}$ (the GC method using the average of $\hat{C}$ ), and the fourth row $\mathrm{GC}_{2}$ (the $\mathrm{GC}$ method using the median of $\hat{C}$ ).

The results for the three tested $H(t)$ are shown in Fig. 2, in which the upper, the middle, and the lower rows represent $\mathrm{LC}, \mathrm{GC}$ using the average of $\hat{C}\left(\mathrm{GC}_{1}\right)$, and $\mathrm{GC}$ using the median of $\hat{C}\left(\mathrm{GC}_{2}\right)$, respectively. It is clear that the results of $\hat{H}(t)$ by GC are more accurate and also preserve sharp confidence intervals when compared with those by LC. The method GC using the median of $\hat{C}$ tends to preserve sharper confidence intervals than that using the average of $\hat{C}$. Regarding estimated $C, \mathrm{GC}$ also produced high accuracy and stability. Figure 3 shows normalized histograms of estimated $C$ for the U-shape $H(t)$ function and the three methods in comparison, in which the GC methods result in more accurate distributions for $C$ than the $\mathrm{LC}$ method. In particular, the average of the $\mathrm{GC}_{2}$ method was very close to the true value of $C=3$, which led to an accurate and stable $H(t)$ estimation, as Fig. $2 \mathrm{~g}$ shows. The overall result is not surprising because GC, adapting the estimator $\hat{C}$ in Eq. (6) at the first phase, caused the estimation of $H(t)$ in Eq. (7) to be more accurate and sharp than the interim estimator $\hat{H}_{L}(t)$ in terms

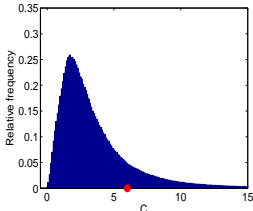

(a) LC

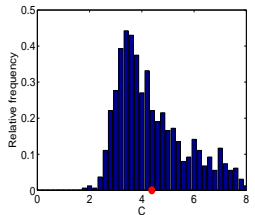

(b) $\mathrm{GC}_{1}$

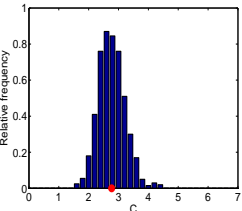

(c) $\mathrm{GC}_{2}$
Fig. 3. Normalized histograms of estimated $C$ for (a) the LC method, (b) the $\mathrm{GC}_{1}$ method, and (c) the $\mathrm{GC}_{2}$ method are shown. The results are based on simulated $\mathrm{mBm}$ signals from the U-shape $H(t)$ and true $C=3$. The red dot represents its average of estimated C.

of the confidence bands in Fig. 2. Additionally, the empirical confidence bands for the flat-line function contain the flat line as in Fig. 2i and 2l, supporting the idea that the signal could be modeled with fBm for simplicity.

To see effects of neighborhood size $\left|v_{t}\right|$ and order $k$ in absolute moments, $\left|v_{t}\right|$ varied from 30 to 150 and 400, and $k$ from 2 to 3 and 4 . We notice that for LC only $k=2$ was available (Coeurjolly, 2005). The results, summarized in Table 2, show that, for U-shape and logistic $H(t)$ and the LC and $\mathrm{GC}_{1}$ methods, MSE reached its minimum at $\left|v_{t}\right|=150$ and then increased as $k$ increased, while for the $\mathrm{GC}_{2}$ method the minimum occurred at $\left|v_{t}\right|=30$. It implies that there is an optimal neighborhood size for the methods depending on signal types, not too small or too large to catch local Hurst exponents out of sample paths. For the flat-line signal, MSE decreased as neighborhood size $\left|v_{t}\right|$ increased, which is reasonable because longer sample paths of a homogeneous regularity level will stabilize the estimation of the regularity level. In most cases for the U-shape and logistic signals and the three methods, MSE increased as $k$ increases, which suggests the use of $k=2$ for a practical setting. Considering a local sample path of $\mathrm{mBm}$ as an approximation of $\mathrm{fBm}$, this empirical observation under $\mathrm{mBm}$ settings coincides with the result in Coeurjolly (2001) that theoretical variance of estimated $H(t)$ under $\mathrm{fBm}$ settings is minimal for $k=2$. It is also found that the GC method consistently outperformed the LC method in MSE.

In addition, Fig. 4 shows trends of averaged MSE for the method of $\mathrm{GC}_{1}$ depending on neighborhood size $\left|v_{t}\right|$ and filter dilation $M$ : the average MSE decreased substantially to the minimum near neighborhood size $\left|v_{t}\right|=100$ for the U-shape and logistic $H(t)$, while the average MSE decreased more rapidly for the flat-line $H(t)$; averaged MSE dropped significantly near filter dilation $M=3$ for all the tested $H(t)$. For practical purposes, a neighborhood size around $\left|v_{t}\right|=100$ and filter dilation $M=3$ are suggested. 
Table 2. MSE averages for different neighborhood sizes $\left(v_{t}\right)$ and the exponent in absolute moments $(k)$ depending on the three test functions through one thousand $\mathrm{mBm}$ series for each case are shown.

\begin{tabular}{|c|c|c|c|c|c|c|c|c|c|c|}
\hline \multirow[b]{2}{*}{$H(t)$ type } & \multirow[t]{2}{*}{$\left|v_{t}\right|$} & \multicolumn{3}{|c|}{$\begin{array}{c}30 \\
k\end{array}$} & \multicolumn{3}{|c|}{$\begin{array}{c}150 \\
k\end{array}$} & \multicolumn{3}{|c|}{$\begin{array}{c}400 \\
k\end{array}$} \\
\hline & & 2 & 3 & 4 & 2 & 3 & 4 & 2 & 3 & 4 \\
\hline \multirow{4}{*}{ U-shape } & WV & 42.5 & - & - & 33.2 & - & - & 38.7 & - & - \\
\hline & LC & 31.6 & - & - & 15.1 & - & - & 13.8 & - & - \\
\hline & $\mathrm{GC}_{1}$ & 32.4 & 34.1 & 32.9 & 4.11 & 5.89 & 7.12 & 10.4 & 13.8 & 16.0 \\
\hline & $\mathrm{GC}_{2}$ & 1.59 & 1.56 & 1.55 & 5.56 & 7.65 & 9.36 & 10.4 & 13.8 & 15.9 \\
\hline \multirow{4}{*}{ logistic } & WV & 43.8 & - & - & 35.9 & - & - & 41.1 & - & - \\
\hline & $\mathrm{LC}$ & 32.6 & - & - & 9.94 & - & - & 44.2 & - & - \\
\hline & $\mathrm{GC}_{1}$ & 57.2 & 59.3 & 61.3 & 2.17 & 3.08 & 3.11 & 23.9 & 31.8 & 37.1 \\
\hline & $\mathrm{GC}_{2}$ & 1.55 & 1.37 & 1.48 & 2.43 & 3.18 & 3.98 & 24.2 & 32.1 & 37.5 \\
\hline \multirow{4}{*}{ flat-line } & WV & 41.6 & - & - & 9.31 & - & - & 4.24 & - & - \\
\hline & $\mathrm{LC}$ & 35.4 & - & - & 5.67 & - & - & 1.53 & - & - \\
\hline & $\mathrm{GC}_{1}$ & 11.3 & 10.8 & 12.1 & 1.12 & 1.08 & 1.08 & 0.608 & 0.655 & 0.648 \\
\hline & $\mathrm{GC}_{2}$ & 1.55 & 1.59 & 1.55 & 1.01 & 0.935 & 1.01 & 0.646 & 0.665 & 0.664 \\
\hline
\end{tabular}

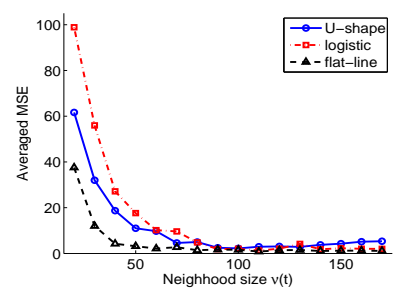

(a)

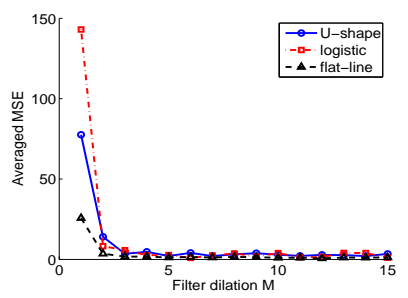

(b)
Fig. 4. MSE averages for $\mathrm{GC}_{1}$ depending on (a) different neighborhood sizes $v_{t}$ and (b) filter dilation $M$ are shown.

\section{Application to turbulence signals}

The proposed method is applied to turbulence signals of air velocities and temperatures. The velocity in longitudinal $\left(u_{1}\right)$, lateral $\left(u_{2}\right)$, and vertical $\left(u_{3}\right)$ directions, and air temperature $(T)$ measurements by a GILL triaxial sonic anemometer were carried out on 12-16 July and 3-6 August 1995, at $5.2 \mathrm{~m}$ above the ground surface over an Alta Fescue grass site at the Blackwood division of the Duke Forest in Durham, North Carolina. During the experiment, maximum mean air temperature up to $38^{\circ} \mathrm{C}$ was measured in Durham. The site is a $480-\mathrm{m}$ by $305-\mathrm{m}$ grass-covered forest clearing $\left(36^{\circ} 2^{\prime} \mathrm{N}, 79^{\circ} 8^{\prime} \mathrm{W}\right.$, elevation $\left.=163 \mathrm{~m}\right)$. Sonic anemometers measure velocity by sensing the effect of wind on transit times of sound pulses traveling in opposite directions across a known instrument distance. The measurements were subsequently divided into $19.5 \mathrm{~min}$ intervals to produce $N=65536$ time measurements per run. Further details about the experimental setup, atmospheric conditions, inertial subrange identification, and instrumentation details can be found in Katul et al. (1997). This paper focuses on 103 runs collected over a wide range of atmospheric stability conditions. Time-changing Hurst exponents $H(t)$ and variance parameters $C$ were estimated to relate to atmospheric stability conditions. Each velocity and temperature run was mean-centered prior to the estimation of $H(t)$ and $C$.

Atmospheric stability was measured by the Obukhov length $(L)$, which is used as an indicator of atmospheric mixing conditions in surface layers, following the MoninObukhov similarity theory (Monin and Obukhov, 1954; Obukhov, 1971; Stull, 1988). The Obukhov length describes a characteristic length scale, i.e. the height of the dynamic sub-layer at the base of the boundary layer (Obukhov, 1971; Schertzer et al., 2011). The sign of the surface heat flux in the Obukhov length indicates whether the boundary layer is statically stable, related to negative buoyancy terms, or statically unstable, related to positive buoyancy terms. Atmospheric stability measure by the Obukhov length can also be found in a lot of recent research (Van den Berg, 2008; Wharton and Lundquist, 2011). Based on the Obukhov length, the 103 runs are classified into stable conditions ( $L>0.05,48$ runs) and unstable conditions ( $L<0.05,55$ runs), as in Katul et al. (2003). Figures 5 and 6 show runs of the two velocity (longitudinal $u_{1}$ and vertical $u_{3}$ ) and temperature signals under stable conditions and unstable conditions in the upper rows, respectively, with estimated $H(t)$ in the lower rows. Seemingly, the velocity signals showed more fluctuation of regularity than the temperature signals, which is consistent with the observation that the scaling of temperature differs from that of velocity (Lovejoy et al., 2009). The sample averages $\left(\operatorname{Avg}_{\hat{H}, s}\right.$ for signal $\left.s\right)$ and the sample variance of estimated $H(t)\left(\operatorname{Var}_{\hat{H}, s}\right.$ for signal $\left.s\right)$ and the estimated $C\left(\hat{C}_{s}\right.$ for signal $s)$ per run were computed. That is to say, $\operatorname{Avg}_{\hat{H}, u_{1}}$ means the 


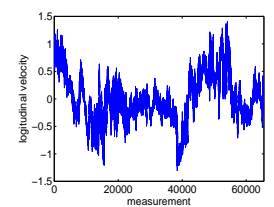

(a) $u_{1}$

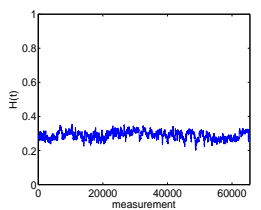

(d) $\hat{H}(t)$ for $u_{1}$

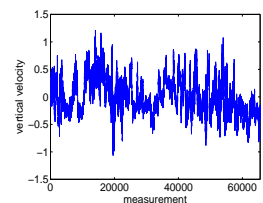

(b) $u_{3}$

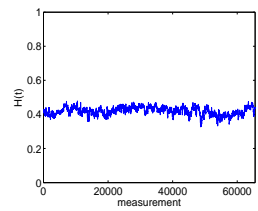

(e) $\hat{H}(t)$ for $u_{3}$

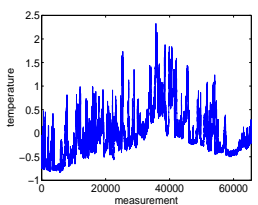

(c) $T$

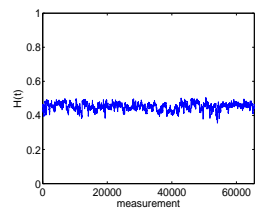

(f) $\hat{H}(t)$ for $T$
Fig. 5. Two velocity (a longitudinal $u_{1}$ and b vertical $u_{3}$ ) and (c) temperature $T$ signals under stable conditions from the 12th run are shown in the upper row. The corresponding estimated $H(t)$ are shown in the lower row.

average of estimated $H(t)$ of longitudinal signal $u_{1}$ with respect to $t$. $\operatorname{Avg}_{\hat{H}, s}$ was adopted as the global Hurst exponent of the whole set of observed $H(t)$, while $\operatorname{Var}_{\hat{H}, s}$ was chosen to represent how much the signal differs from $\mathrm{fBm}$ models. The estimated $C$ itself represents the energy level of the signal.

Table 3 shows the averages of those descriptive statistics for stable runs and unstable runs. For instance, the variability of $\hat{H}$ on average for $u_{3}$ from the unstable runs $(0.0313)$ was larger than that for $T(0.0240)$ as is illustrated in Fig. 6e and $\mathrm{f}$. In addition, the two groups of stable and unstable runs were compared, and the significance for the group difference was presented for each signal $s$ and descriptive statistic. Among those significant with significance level 0.05 are $\operatorname{Avg}_{\hat{H}, T}$, $\operatorname{Var}_{\hat{H}, u_{2}}, \operatorname{Var}_{\hat{H}, u_{3}}$, and $\hat{C}_{T}$. In other words, velocity signals exhibited separation of stable and unstable runs in fluctuations of regularity, and temperature signals distinguished stable runs from unstable runs by either global regularity levels or energy levels.

To classify atmospheric stability conditions using $\mathrm{mBm}$ models, the quality of those descriptors from $\mathrm{mBm}$ models was measured with classification functions, linear discriminant analysis (LDA), quadratic discriminant analysis (QDA), and support vector machines (SVMs with radial basis function and width parameter 1), which separate stable runs from unstable ones. A training set of 73 randomly chosen runs $(70 \%)$ out of the total 103 runs and a testing set of the remaining 32 runs $(30 \%)$ were generated. Then by each of the classification methods a correct classification rate for the testing set was computed. This procedure was iterated 5000 times, producing the average of the correct classification rates for each method. In addition to the proposed method GC using the mean for $\hat{C}$, two other methods, WV and $\mathrm{LC}$, were also included for the sake of comparison.

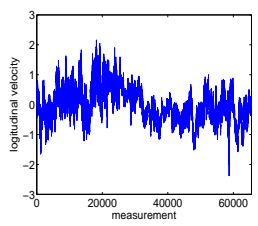

(a) $u_{1}$

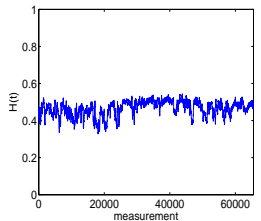

(d) $\hat{H}(t)$ for $u_{1}$

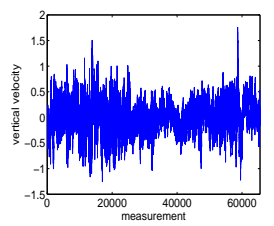

(b) $u_{3}$

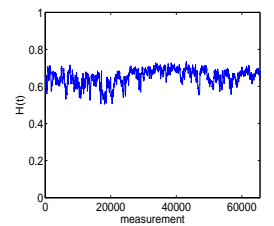

(e) $\hat{H}(t)$ for $u_{3}$

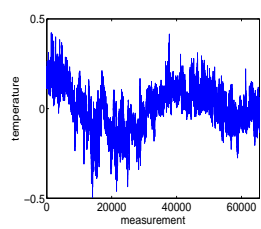

(c) $T$

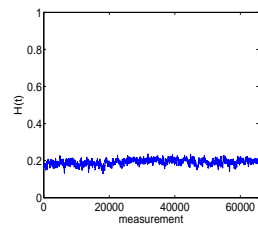

(f) $\hat{H}(t)$ for $T$
Fig. 6. Two velocity (a longitudinal $u_{1}$ and b vertical $u_{3}$ ) and (c) temperature $T$ signals under unstable conditions from the 7 th run are shown in the upper row. The corresponding estimated $H(t)$ are shown in the lower row.

Out of all possible combinations, that is to say $2^{12}-1$ combinations due to 3 descriptors $\left(\operatorname{Avg}_{\hat{H}, s}, \operatorname{Var}_{\hat{H}, s}, \hat{C}_{s}\right)$ and 4 signals $\left(s=u_{1}, u_{2}, u_{3}, T\right)$, each individual descriptor is compared first. Table 4 shows classification rates for each individual descriptor, indicating $\operatorname{Avg}_{\hat{H}, T}$ is one single descriptor for atmospheric stability conditions. As a baseline for comparison, when signals are directly classified by themselves through the $\mathrm{k}$-nearest-neighborhood method $(\mathrm{k}=7)$ instead of using descriptors, the classification rates were $0.506,0.494,0.496$, and 0.495 for each of velocity and temperature signals. Additionally, a descriptor set of $\operatorname{Avg}_{\hat{H}, s}$, $\operatorname{Var}_{\hat{H}, s}$, and $\hat{C}_{s}$ for all velocity and temperature signals $(s=$ $\left.u_{1}, u_{2}, u_{3}, T\right)$ resulted in classification rate 0.617 for LDA; for $\operatorname{Avg}_{\hat{H}, s}$ and $\hat{C}_{s}, 0.613$; for $\operatorname{Var}_{\hat{H}, s}$ and $\hat{C}_{s}, 0.576$; and for $\operatorname{Avg}_{\hat{H}, s}$ and $\operatorname{Var}_{\hat{H}, s}, 0.655$. It was found that descriptors $\operatorname{Avg}_{\hat{H}, s}$ and $\operatorname{Var}_{\hat{H}, s}$ were important for atmospheric stability conditions while $\hat{C}_{s}$ was not. Furthermore, through search of classification rates by each combination of descriptors, the following three sets of descriptors were chosen as important ones that have high classification rates: $\operatorname{Var}_{\hat{H}, u_{1}}$ and $\operatorname{Avg}_{\hat{H}, T}$; $\operatorname{Var}_{\hat{H}, u_{2}}$ and $\operatorname{Avg}_{\hat{H}, T} ;$ and $\operatorname{Var}_{\hat{H}, u_{3}}$ and $\operatorname{Avg}_{\hat{H}, T}$, as shown in Table 5. Additionally, the classification rates by GC turned out to be consistently higher than those by the other methods. This result shows that overall regularity levels of a temperature signal and fluctuations of regularity levels of a velocity signal are important $\mathrm{mBm}$-model driven descriptors that account for atmospheric stability conditions.

\section{Conclusions}

For the study of atmospheric turbulence in nonlinear geophysics, a generalization of fractional Brownian motions, multifractional Brownian motions $(\mathrm{mBm})$ as well as multifractality and generalized scale invariance analysis are 
Table 3. Summary of the sample averages and variances for three velocity and temperature signals in terms of average and $p$ value for stable and unstable runs using two-sample t-tests is shown. The boldfaced numbers represent significance with significance level 0.05 .

\begin{tabular}{|c|c|c|c|c|c|c|c|c|c|}
\hline \multirow[b]{2}{*}{$s$} & \multicolumn{3}{|c|}{$\operatorname{Avg}_{\hat{H}, s}$} & \multicolumn{3}{|c|}{$\operatorname{Var}_{\hat{H}, s}$} & \multicolumn{3}{|c|}{$\hat{C}_{s}$} \\
\hline & stable & unstable & $p$ value & stable & unstable & $p$ value & stable & unstable & $p$ value \\
\hline$u_{1}$ & 0.407 & 0.423 & 0.358 & 0.0276 & 0.0294 & 0.074 & 11.1 & 17.3 & 0.103 \\
\hline$u_{2}$ & 0.467 & 0.479 & 0.528 & 0.0274 & 0.0297 & 0.045 & 24.4 & 33.7 & 0.175 \\
\hline$u_{3}$ & 0.608 & 0.610 & 0.918 & 0.0285 & 0.0313 & 0.039 & 85.9 & 99.1 & 0.374 \\
\hline$T$ & 0.375 & 0.268 & $<\mathbf{0 . 0 0 0}$ & 0.0237 & 0.0240 & 0.820 & 8.25 & 3.33 & 0.032 \\
\hline
\end{tabular}

Table 4. Average correct classification rates through five thousands partitioning of the total 103 runs into training (70\%) and testing (30\%) sets are shown for the classification methods (LDA, QDA, SVM), the estimation methods (WV, LC, GC), and each individual descriptor.

\begin{tabular}{|c|c|c|c|c|c|c|c|}
\hline & & \multicolumn{6}{|c|}{ individual descriptor } \\
\hline & & \multicolumn{3}{|c|}{$u_{1}$} & \multicolumn{3}{|c|}{$u_{2}$} \\
\hline & & $\operatorname{Var}_{\hat{H}, u_{1}}$ & $\operatorname{Avg}_{\hat{H}, u_{1}}$ & $\hat{C}_{u_{1}}$ & $\operatorname{Var}_{\hat{H}, u_{2}}$ & $\operatorname{Avg}_{\hat{H}, u_{2}}$ & $\hat{C}_{u_{2}}$ \\
\hline \multirow{3}{*}{ LDA } & WV & 0.542 & 0.613 & 0.482 & 0.543 & 0.579 & 0.518 \\
\hline & $\mathrm{LC}$ & 0.540 & 0.554 & 0.584 & 0.543 & 0.537 & 0.526 \\
\hline & $\mathrm{GC}$ & 0.541 & 0.554 & 0.563 & 0.543 & 0.541 & 0.592 \\
\hline \multirow{3}{*}{ QDA } & WV & 0.550 & 0.617 & 0.536 & 0.558 & 0.588 & 0.508 \\
\hline & $\mathrm{LC}$ & 0.549 & 0.557 & 0.569 & 0.559 & 0.546 & 0.533 \\
\hline & $\mathrm{GC}$ & 0.549 & 0.553 & 0.562 & 0.559 & 0.550 & 0.578 \\
\hline \multirow{5}{*}{ SVM } & WV & 0.461 & 0.542 & 0.506 & 0.442 & 0.575 & 0.442 \\
\hline & LC & 0.464 & 0.496 & 0.555 & 0.444 & 0.471 & 0.488 \\
\hline & $\mathrm{GC}$ & 0.463 & 0.491 & 0.490 & 0.446 & 0.473 & 0.502 \\
\hline & & \multicolumn{3}{|c|}{$u_{3}$} & \multicolumn{3}{|c|}{$T$} \\
\hline & & $\operatorname{Var}_{\hat{H}, u_{3}}$ & $\operatorname{Avg}_{\hat{H}, u_{3}}$ & $\hat{C}_{u_{3}}$ & $\operatorname{Var}_{\hat{H}, T}$ & $\operatorname{Avg}_{\hat{H}, T}$ & $\hat{C}_{T}$ \\
\hline \multirow{3}{*}{ LDA } & WV & 0.501 & 0.619 & 0.516 & 0.588 & 0.614 & 0.524 \\
\hline & $\mathrm{LC}$ & 0.502 & 0.525 & 0.540 & 0.588 & 0.638 & 0.683 \\
\hline & $\mathrm{GC}$ & 0.502 & 0.526 & 0.577 & 0.588 & 0.657 & 0.529 \\
\hline \multirow{3}{*}{ QDA } & WV & 0.533 & 0.632 & 0.551 & 0.561 & 0.639 & 0.551 \\
\hline & LC & 0.533 & 0.544 & 0.542 & 0.561 & 0.641 & 0.666 \\
\hline & $\mathrm{GC}$ & 0.533 & 0.540 & 0.608 & 0.562 & 0.658 & 0.598 \\
\hline \multirow{3}{*}{ SVM } & WV & 0.476 & 0.551 & 0.527 & 0.654 & 0.573 & 0.531 \\
\hline & $\mathrm{LC}$ & 0.474 & 0.572 & 0.460 & 0.654 & 0.647 & 0.657 \\
\hline & $\mathrm{GC}$ & 0.472 & 0.578 & 0.563 & 0.658 & 0.654 & 0.562 \\
\hline
\end{tabular}

commonly used. This paper discusses the identification of the time-changing Hurst exponent $H(t)$ and variance level $C$ of a sampled path of a process from the multifractional Brownian model and its application to turbulence signals. It proposes a two-phased approach to estimate $H(t)$ and $C$ that relates the $k$-th absolute moments to the $k$-th empirical moments after filtering a sampled path. It takes advantage of accurate estimation of $C$, which is unvarying regardless of any position in the sampled path, in the first phase and improves estimation of $H(t)$ using the exact nonlinear moment relationship in the second phase. The performance of the proposed method is demonstrated in a simulation study in which several types of known $H(t)$ and $C$ are estimated from simulated $\mathrm{mBm}$ signals in comparison to a selected previous method. It produced more accurate and sharp estimation of $H(t)$ and $C$. The method was applied to air turbulence signals to identify time-changing regularity and global variability and to associate them with atmospheric stability. The analysis of 103 runs show that velocity signals show differences of stable and unstable runs in fluctuations of regularity, while temperature signals distinguished the two kinds by stable behavior of regularity and energy levels. It also shows that regularity 
Table 5. Average correct classification rates through five thousand partitions of the total 103 runs into training (70\%) and testing (30\%) sets are shown for the classification methods (LDA, QDA, SVM), the estimation methods (WV, LC, GC), and the chosen descriptors.

\begin{tabular}{ccccc}
\hline \multicolumn{4}{c}{ chosen descriptors } \\
\hline & & $\operatorname{Var}_{\hat{H}, u_{1}}, \operatorname{Avg}_{\hat{H}, T}$ & $\operatorname{Var}_{\hat{H}, u_{2}}, \operatorname{Avg}_{\hat{H}, T}$ & $\operatorname{Var}_{\hat{H}, u_{3}}, \operatorname{Avg}_{\hat{H}, T}$ \\
\hline \multirow{2}{*}{ LDA } & WV & 0.632 & 0.618 & 0.620 \\
& LC & 0.642 & 0.656 & 0.639 \\
& GC & $\mathbf{0 . 7 1 3}$ & $\mathbf{0 . 7 1 7}$ & $\mathbf{0 . 7 1 9}$ \\
\hline \multirow{5}{*}{ QDA } & WV & 0.644 & 0.633 & 0.642 \\
& LC & 0.645 & 0.668 & 0.647 \\
& GC & $\mathbf{0 . 7 1 8}$ & $\mathbf{0 . 7 1 4}$ & $\mathbf{0 . 7 2 4}$ \\
\hline \multirow{3}{*}{ SVM } & WV & 0.561 & 0.568 & 0.624 \\
& LC & 0.603 & 0.599 & 0.625 \\
& GC & $\mathbf{0 . 6 7 9}$ & $\mathbf{0 . 6 9 0}$ & $\mathbf{0 . 6 8 3}$ \\
\hline
\end{tabular}

fluctuations of a velocity signal and global regularity levels of a temperature signal are important $\mathrm{mBm}$-model driven descriptors that account for atmospheric stability. This study in the context of atmospheric stability can be extended to multifractal analysis capturing rich information on regularity. The suggested method can further investigate the finite-sample bias effect in estimating $H(t)$ with asymptotic results and also can be generalized to handle unequal data points that frequently arise due to missing observations.

\section{Appendix A}

It is enough to show the filtered signal $V_{\mathbf{a}}\left(\frac{j}{N}\right)$ is weakly stationary because $\mathbf{a}$ is replaceable with $\mathbf{a}^{(m)}$. To make $W(t)$ in the first equation of Sect. 2 well defined, let us assume the Hurst exponent $H(t)$ is a Hölderian of order $0<\eta \leq$ 1 on $[0,1], H \in \mathbb{C}^{\eta}([0,1])$ as in Cohen (1999). Let $v(t)$ be an index set of a neighborhood of $t$, defined as $v(t)=$ $\{j \in \mathbb{Z}|l<j \leq N| j / N-t \mid, \leq \epsilon\}$ for a parameter $\epsilon>0$. We set $\epsilon$ to be a function of $N$ in such a way that $\epsilon \rightarrow 0$, $\epsilon N \rightarrow \infty$, and $\epsilon^{\eta} \log (N) \rightarrow 0$ as $N \rightarrow \infty$. In other words, for a sufficiently large $N$, the size of a neighborhood becomes sufficiently small while maintaining the summation of the sizes of all neighborhoods sufficiently large. Let $G(t)$ denote $C \sqrt{K(2 H(t)) / 2}$ for simplicity.

For $j_{1} / N, j_{2} / N \in v(t)$, the covariance $\mathcal{E}\left[V_{\mathbf{a}}\left(\frac{j_{1}}{N}\right) V_{\mathbf{a}}\left(\frac{j_{2}}{N}\right)\right]$ becomes, by $u=\frac{\lambda}{N}$,

$$
\begin{aligned}
\sum_{q_{1}, q_{2}} a_{q_{1}} a_{q_{2}}( & G\left(\frac{j_{1}-q_{1}}{N}\right) G\left(\frac{j_{2}-q_{2}}{N}\right) \int \frac{\exp \left(\mathbf{i} \frac{j_{1}-q_{1}}{N} \lambda\right)-1}{|\lambda|^{H\left(\frac{j_{1}-q_{1}}{N}\right)+1 / 2}} \\
\times & \frac{\exp \left(-\mathbf{i} \frac{j_{2}-q_{2}}{N} \lambda\right)-1}{\left.|\lambda|^{H\left(\frac{j_{2}-q_{2}}{N}\right)+1 / 2} d \lambda\right)}
\end{aligned}
$$

$$
\begin{gathered}
=\sum_{q_{1}, q_{2}} a_{q_{1}} a_{q_{2}} \frac{G\left(\frac{j_{1}-q_{1}}{N}\right) G\left(\frac{j_{2}-q_{2}}{N}\right)}{N^{H\left(\frac{j_{1}-q_{1}}{N}\right)+H\left(\frac{j_{2}-q_{2}}{N}\right)}} \int \frac{\exp \left(\mathbf{i}\left(j_{1}-q_{1}\right) u\right)-1}{|u|^{H\left(\frac{j_{1}-q_{1}}{N}\right)+1 / 2}} \\
\times \frac{\exp \left(-\mathbf{i}\left(j_{2}-q_{2}\right) u\right)-1}{|u|^{H\left(\frac{j_{2}-q_{2}}{N}\right)+1 / 2}} d u .
\end{gathered}
$$

By Taylor's expansion and a Hölderian order $\eta$ of $H(t)$, we approximate

$$
\begin{aligned}
& \frac{1}{N^{H\left(\frac{j-q}{N}\right)}}=\frac{1}{N^{H(t)}}\left(1+\mathcal{O}\left(\epsilon^{\eta} \log N\right)\right), \\
& \frac{1}{|u|^{H\left(\frac{j-q}{N}\right)}}=\frac{1}{|u|^{H(t)}}\left(1+\mathcal{O}\left(\epsilon^{\eta} \log u\right)\right), \\
& G\left(\frac{j-q}{N}\right)=G(t)+\mathcal{O}\left(\epsilon^{\eta}\right) .
\end{aligned}
$$

Using also $\epsilon^{\eta} \rightarrow 0$ and $\epsilon^{\eta} \log (N) \rightarrow 0$ as $N$ goes to infinity, the covariance becomes

$$
\begin{aligned}
\sum_{q_{1}, q_{2}} a_{q_{1}} a_{q_{2}} & \frac{G^{2}(t)}{N^{2 H(t)}} \int \frac{\exp \left(\mathbf{i}\left(j_{1}-q_{1}\right) u\right)-1}{|u|^{H(t)+1 / 2}} \\
& \times \frac{\exp \left(-\mathbf{i}\left(j_{2}-q_{2}\right) u\right)-1}{|u|^{H(t)+1 / 2}} d u .
\end{aligned}
$$

By the vanishing-moments property of $\mathbf{a}$, the above equation is simplified:

$$
\sum_{q_{1}, q_{2}} a_{q_{1}} a_{q_{2}} \frac{G^{2}(t)}{N^{2 H(t)}} \int \frac{\cos \left(\left(j_{2}-j_{1}+q_{1}-q_{2}\right) u\right)-1}{|u|^{2 H(t)+1}} d u .
$$


Since $K(\alpha) \int \frac{1-\cos (\kappa u)}{|u|^{\alpha+1}} d u=|\kappa|^{\alpha}, \forall \kappa$, and $0<\alpha<2$, the covariance is rewritten:

$$
\begin{aligned}
\mathcal{E} & {\left[V_{\mathbf{a}}\left(\frac{j_{1}}{N}\right) V_{\mathbf{a}}\left(\frac{j_{2}}{N}\right)\right]=} \\
& -\sum_{q_{1}, q_{2}} a_{q_{1}} a_{q_{2}} \frac{G^{2}(t)}{N^{2 H(t)}} \frac{\left|j_{2}-j_{1}+q_{1}-q_{2}\right|^{2 H(t)}}{K(2 H(t))} \\
& =-\frac{C^{2}}{N^{2 H(t)}} \sum_{q_{1}, q_{2}} \frac{a_{q_{1}} a_{q_{2}}}{2}\left|j_{2}-j_{1}+q_{1}-q_{2}\right|^{2 H(t)} .
\end{aligned}
$$

Consequently, the covariance $\mathcal{E}\left[V_{\mathbf{a}}\left(\frac{j_{1}}{N}\right) V_{\mathbf{a}}\left(\frac{j_{2}}{N}\right)\right]$ depends on $j_{1}-j_{2}$, which makes the filtered signal $V_{\mathbf{a}}\left(\frac{j}{N}\right)$ weakly stationary. In addition, when $j_{1}$ and $j_{2}$ are identical with $j$, $\operatorname{Var}\left[V_{\mathbf{a}}\left(\frac{j}{N}\right)\right]=\frac{C^{2}}{N^{2 H(t)}} \pi_{\mathbf{a}, H(t)}$. When a is replaced with $\mathbf{a}^{(m)}$, $\operatorname{Var}\left[V_{\mathbf{a}^{(m)}}\left(\frac{j}{N}\right)\right]=\frac{C^{2} m^{2 H(t)}}{N^{2 H(t)}} \pi_{\mathbf{a}, H(t)}$.

Acknowledgements. This research was supported by a grant (201200000000605) funded by Hanyang University (HY-2012-N).

Edited by: S. Gaci

Reviewed by: three anonymous referees

\section{References}

Ayache, A., Cohen, S., and Véhel, J.: The covariance structure of multifractional Brownian motion, with application to long range dependence, in: Acoustics, Speech, and Signal Processing, 2000, ICASSP'00, Proceedings 2000 IEEE International Conference, IEEE, 6, 3810-3813, 2000.

Bardet, J. and Bertrand, P.: Definition, properties and wavelet analysis of multiscale fractional Brownian motion, Fractals, 15, 7387, 2007.

Benassi, A., Cohen, S., and Istas, J.: Identifying the multifractional function of a Gaussian process, Statistics Probability Lett., 39, 337-345, 1998.

Benassi, A., Bertrand, P., Cohen, S., and Istas, J.: Identification of the Hurst index of a step fractional Brownian motion, Statist. Inf. Stochastic Process., 3, 101-111, 2000.

Beran, J.: Statistics for long-memory processes, vol. 61, Chapman \& Hall/CRC, 1994.

Coeurjolly, J.: Estimating the parameters of a fractional Brownian motion by discrete variations of its sample paths, Stat. Inf. Stochastic Process., 4, 199-227, 2001.

Coeurjolly, J.: Identification of multifractional Brownian motion, Bernoulli, 11, 987-1008, 2005.

Cohen, S.: From self-similarity to local self-similarity: the estimation problem, Fractals, Theory and Applications in Engineering, Springer, London, 1999.

Daubechies, I.: Ten lectures on wavelets, vol. 61, Society for Industrial Mathematics, 1992.

Fhima, M., Guillin, A., and Bertrand, P.: Fast change point analysis on the Hurst index of piecewise fractional Brownian motion, Journée de Statistiques, arXiv:1103.4029, 2011.
Gao, J.: Multiscale analysis of complex time series: integration of chaos and random fractal theory, and beyond, John Wiley \& Sons, 2007.

Ghil, M.: Turbulence and predictability in geophysical fluid dynamics and climate dynamics, vol. 88, North Holland, 1985.

Katul, G., Hsieh, C., and Sigmon, J.: Energy-inertial scale interactions for velocity and temperature in the unstable atmospheric surface layer, Bound.-Lay. Meteorol., 82, 49-80, 1997.

Katul, G., Angelini, C., De Canditiis, D., Amato, U., Vidakovic, B., and Albertson, J.: Are the effects of large scale flow conditions really lost through the turbulent cascade, Geophys. Res. Lett., 30, 1164, doi:10.1029/2002GL015284, 2003.

Lévy-Véhel, J. and Peltier, R.: Multifractional Brownian motion: definition and preliminary results, Rapport de recherche de l'INRIA n2645, 1995.

Lovejoy, S., Tuck, A., Hovde, S., and Schertzer, D.: Is isotropic turbulence relevant in the atmosphere?, Geophys. Res. Lett., 34, L15802, doi:10.1029/2007GL029359, 2007.

Lovejoy, S., Tuck, A., Hovde, S., and Schertzer, D.: Vertical cascade structure of the atmosphere and multifractal dropsonde outages, J. Geophys. Res., 114, D07111, doi:10.1029/2008JD010651, 2009.

Lovejoy, S., Tuck, A., and Schertzer, D.: Horizontal cascade structure of atmospheric fields determined from aircraft data, J. Geophys. Res., 115, D13105, doi:10.1029/2009JD013353, 2010.

Monin, A. and Obukhov, A.: Basic laws of turbulent mixing in the surface layer of the atmosphere, Trudy Geofiz. Inst. Acad. Nauk SSSR, 24, 163-187, 1954.

Obukhov, A.: Turbulence in an atmosphere with a non-uniform temperature, Bound.-Lay. Meteorol., 2, 7-29, 1971.

Papoulis, A.: Probability, Random Variables, and Stochastic Processes, vol. 3, McGraw-hill New York, 1991.

Schertzer, D. and Lovejoy, S.: Multifractals, generalized scale invariance and complexity in geophysics, Int. J. Bifur. Chaos, 21, 3417-3456, 2011.

Schertzer, D., Tchiguirinskaia, I., Lovejoy, S., and Tuck, A. F.: Quasi-geostrophic turbulence and generalized scale invariance, a theoretical reply, Atmos. Chem. Phys., 12, 327-336, doi:10.5194/acp-12-327-2012, 2012.

Stull, R.: An introduction to boundary layer meteorology, vol. 13, Springer, 1988.

Tuck, A.: From molecules to meteorology via turbulent scale invariance, Q. J. Roy. Meteorol. Soc., 136, 1125-1144, 2010.

Van den Berg, G.: Wind turbine power and sound in relation to atmospheric stability, Wind Energy, 11, 151-169, 2008.

Vidakovic, B.: Statistical modeling by wavelets, Wiley Online Library, 1999.

Wanliss, J. and Dobias, P.: Space storm as a phase transition, J. Atmos. Sol.-Terrest. Phys., 69, 675-684, 2007.

Wharton, S. and Lundquist, J.: Assessing atmospheric stability and its impacts on rotor-disk wind characteristics at an onshore wind farm, Wind Energy, 2011.

Wood, A. and Chan, G.: Simulation of stationary Gaussian processes in $[0,1]$ d, J. Computat. Graphic. Stat., 3, 409-432, 1994. 